\section{Association of non-alcoholic fatty liver disease and polycystic ovarian syndrome}

To cite: Asfari MM, Sarmini MT, Baidoun $\mathrm{F}$, et al. Association of non-alcoholic fatty liver disease and polycystic ovarian syndrome. BMJ Open Gastro 2020;7:e000352. doi:10.1136/ bmjgast-2019-000352

- Additional material is published online only. To view please visit the journal online (http://dx.doi.org/10.1136/ bmjgast-2019-000352).

MMA and MTS contributed equally.

MMA and MTS are joint first authors.

The abstract of this study was presented in the AASLD conference and has been published online at https:// aasldpubs.onlinelibrary.wiley. com/doi/full/10.1002/hep. 30257 .

Received 6 November 2019

Revised 26 May 2020

Accepted 28 May 2020

\section{Check for updates}

(c) Author(s) (or their employer(s)) 2020. Re-use permitted under CC BY-NC. No commercial re-use. See rights and permissions. Published by BMJ.

For numbered affiliations see end of article.

Correspondence to Dr Mohammad Maysara Asfari; mma-86@hotmail.com

\section{ABSTRACT}

Background Polycystic ovarian syndrome (PCOS) is a common endocrine disorder in women. Women with PCOS have androgen excess as a defining feature. They also have increased insulin resistance and obesity, which are also risk factors for non-alcoholic fatty liver disease (NAFLD). However, published data regarding PCOS as independent risk factor for NAFLD remain controversial. Therefore, we conducted this study to evaluate the association between PCOS and NAFLD using a large national database.

Methods We identified adult female patients $(\geq 18$ years) with PCOS using the National Inpatient Sample database between 2002 and 2014. The control group included patients who did not have a diagnosis of PCOS. Multivariate logistic regression analysis was performed to study the association of NAFLD with PCOS.

Results Out of a total of 50785354 women, 77415 $(0.15 \%)$ had PCOS. These patients were younger $(32.7$ vs $54.8 ; p<0.001)$ and more likely to be obese $(29.4 \%$ vs $8.6 \% ; p<0.001$ ) compared with non-PCOS patients. However, the PCOS group had less hypertension (23.2\% vs $39.8 \%$ ), dyslipidaemia (12\% vs $17.8 \%$ ) and diabetes mellitus $(18.1 \%$ vs $18.3 \%)(p<0.001$ for all). Using multivariate logistic regression, patients with PCOS had significantly higher rate of NAFLD (OR $4.30,95 \% \mathrm{Cl} 4.11$ to $4.50, p<0.001$ )

Conclusion Our study showed that patients with PCOS have four times higher risk of developing NAFLD compared with women without PCOS. Further studies are needed to assess if specific PCOS treatments can affect NAFLD progression.

\section{INTRODUCTION}

Non-alcoholic fatty liver disease (NAFLD) is the most common chronic liver disease and is associated with significant morbidity and mortality in the USA. ${ }^{1}$ The prevalence of NAFLD has been increasing dramatically worldwide, particularly in Western countries. ${ }^{1}$ Although NAFLD can progress from accumulation of fat alone (steatosis) without inflammation, to necroinflammation (steatohepatitis), to cirrhosis, along with the sequelae of portal hypertension, majority of patients with NAFLD are asymptomatic. ${ }^{2}$
Summary box

What is already known about this subject?

- Previous studies suggested that polycystic ovarian syndrome (PCOS) is an independent risk factor for non-alcoholic fatty liver disease (NAFLD), but these studies were limited in size.

What are the new findings?

- We conducted a very large cross-sectional study and found that patients with PCOS have increased risk of NAFLD.

How might it impact on clinical practice in the foreseeable future?

- Our study adds to the discussion about the role of PCOS in NAFLD formation.

Polycystic ovarian syndrome (PCOS), also known as hyperandrogenic anovulation (HA), is one of the most common endocrine disorders in women of reproductive age. PCOS affects at least $6 \%$ of women in the USA based on the National Institutes of Health diagnostic criteria. ${ }^{3}$ The three essential components of PCOS are hyperandrogenism, ovulatory dysfunction and/ or ultrasound findings of polycystic ovarian morphology. ${ }^{4}$

The major risk factors for NAFLD are obesity, diabetes mellitus (DM), dyslipidaemia and metabolic syndrome. These factors are also common comorbidities in patients with PCOS, ${ }^{15} 6$ which raises the question of a possible association between PCOS and metabolic syndrome. Multiple studies have shown an association between PCOS and NAFLD, with possible hyperandrogenism being an independent risk factor for NAFLD. ${ }^{2} 78$ However, other studies have pointed that hyperandrogenism does not increase the risk of NAFLD. ${ }^{9}$

Hence, the purposes of this study were to investigate the association between NAFLD 
and PCOS and to determine if PCOS is more associated with the development of NAFLD.

\section{METHODS}

\section{Patient population}

This cross-sectional study was conducted using the National Inpatient Sample (NIS) data from 2002 to 2014. The NIS is the largest all-payer inpatient database in the USA and contains a sample of over eight million inpatient hospitalisations each year, which represents approximately $20 \%$ of all discharges from all community hospitals participating in the Healthcare Cost and Utilization Project (HCUP). It excludes rehabilitation and long-term acute care hospitals. Each record of the NIS data includes primary and secondary diagnoses up to 25 and primary and secondary procedures up to 15 . It also contains patient demographics, discharge status, length of stay, disease severity, and comorbidity measures.

\section{Study population, inclusion, and exclusion criteria}

All adult female patients ( $\geq 18$ years old) were included from the NIS during the years 2002-2014. Using the International Classification of Diseases Ninth Version (ICD-9) code, we identified all records with PCOS and NAFLD using the following codes: 256.4 and 571.8, respectively. Patient demographics and comorbidities were identified using the Clinical Classifications Software codes provided by the HCUP, the Elixhauser comorbidities and appropriate ICD-9 codes. Online supplementary tables 1 and 2 show the ICD-9 codes and Elixhauser comorbidities used for PCOS, NAFLD and other comorbidities of interest.

To assess the association between PCOS and NAFLD, we divided patients into two groups: women with PCOS (study group) and women without PCOS (control group). We computed a univariable and a multivariable logistic regression analysis comparing the two groups together after adjusting for potential confounding factors.

\section{Statistical analysis}

Data are expressed as mean $\pm \mathrm{SD}$, and frequencies are reported in percentages. Independent t-tests were used for comparison of continuous variable measurements and $\chi^{2}$ test for categorical variables. Multiple logistic regression was used to assess the association between PCOS and NAFLD. The regression model was adjusted for patients' age, race, gender, relevant comorbidities (hypertension, dyslipidaemia, obesity, hypothyroidism and DM), hospital location, patient medical insurance and socioeconomic status. $\mathrm{P}$ values less than 0.05 were considered statistically significant. SPSS V.25 software was used for all statistical analyses.

\section{RESULTS}

Our nationwide cohort evaluated a total of 50785354 female hospital stays, of whom $77415(0.15 \%)$ had PCOS. As shown in table 1 , patients with PCOS were younger (32.9 vs 54.8) and were less likely to be African-American
Table 1 Comparison of baseline characteristics between PCOS and non-PCOS patients

\begin{tabular}{|c|c|c|c|}
\hline Variable & Pcos & Non-PCOS & $P$ value \\
\hline Age $($ mean $\pm S D)$ & $32.97 \pm 8.70$ & $54.83 \pm 22.31$ & $<0.001$ \\
\hline Race (\%) & & & $<0.001$ \\
\hline White & 75.0 & 67.2 & \\
\hline Black & 10.6 & 14.5 & \\
\hline Hispanic & 8.7 & 11.9 & \\
\hline $\begin{array}{l}\text { Asian or Pacific } \\
\text { Islander }\end{array}$ & 2.1 & 2.6 & \\
\hline Native American & 0.6 & 0.6 & \\
\hline Other & 3.0 & 3.0 & \\
\hline Hospital region (\%) & & & $<0.001$ \\
\hline Northeast & 18.5 & 18.9 & \\
\hline Midwest & 26.6 & 22.8 & \\
\hline South & 35.8 & 39.3 & \\
\hline West & 19.1 & 19.0 & \\
\hline Obesity (\%) & 29.4 & 8.6 & $<0.001$ \\
\hline Hypertension (\%) & 23.2 & 39.83 & $<0.001$ \\
\hline Dyslipidaemia (\%) & 16.1 & 15.1 & $<0.001$ \\
\hline $\begin{array}{l}\text { Diabetes mellitus } \\
(\%)\end{array}$ & 18.1 & 18.3 & $<0.001$ \\
\hline Hypothyroidism (\%) & 12.2 & 11.8 & $<0.001$ \\
\hline
\end{tabular}

PCOS, polycystic ovarian syndrome.

(10.6\% vs $14.6 \%$ ) compared with patients without PCOS ( $\mathrm{p}<0.001$ for all). Patients in the PCOS group also had a higher prevalence of obesity (29.4\% vs $8.6 \%)$ and hypothyroidism $(12.2 \%$ vs $11.8 \%)$ compared with women without PCOS ( $p<0.001$ for all). However, patients in the PCOS group had a lower prevalence of DM $(18.1 \%$ vs $18.3 \%)$, dyslipidaemia (12\% vs $17.8 \%$ ) and hypertension $(23.2 \%$ vs $39.8 \%) \quad(p<0.001$ for all $)$ compared with the control group.

Using univariable logistic regression for the association between PCOS and NAFLD, patients with PCOS had almost eight times higher odds of having NAFLD (OR 7.86, $95 \%$ CI 7.54 to $8.18, \mathrm{p}<0.001)$. After adjusting for possible confounding factors including patients' age, race, gender, hospital location, medical insurance and socioeconomic status, and other relevant comorbidities (obesity, hypertension, dyslipidaemia, DM and hypothyroidism), patients in the PCOS group were still more likely to have NAFLD compared with patients in the control group (OR 4.30, 95\% CI 4.11 to $4.50, \mathrm{p}<0.001$ ) (figure 1). Multivariate regression analysis is shown in table 2.

\section{DISCUSSION}

This analysis of a large nationwide data set demonstrates that women with PCOS are four times more likely to have NAFLD compared with patients without PCOS after 


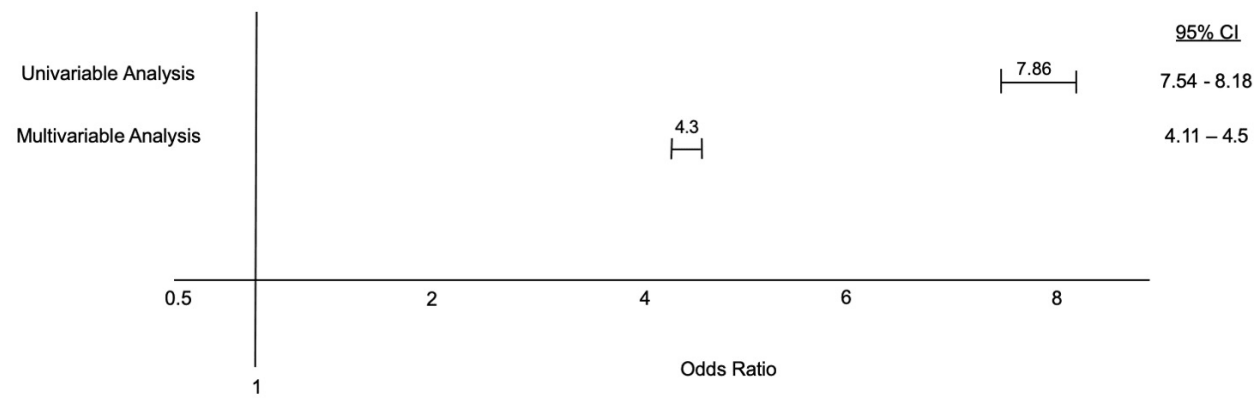

Figure 1 Association between polycystic ovarian syndrome and non-alcoholic fatty liver disease.

adjusting for confounding factors such as obesity and DM.

NAFLD is the most common chronic liver disease in the USA and in the Western world. ${ }^{10}$ Patients with NAFLD may have a wide spectrum of histological findings, ranging from simple steatosis to non-alcoholic steatohepatitis (NASH) (which is characterised by steatosis along with varying degrees of ballooning, inflammation and fibrosis), and ultimately cirrhosis.

Women with PCOS, and similarly with NAFLD, have higher rates of metabolic syndrome and risk factors, including insulin resistance (IR), obesity, hyperlipidaemia and hypertension. ${ }^{11} 12$ Multiple studies have evaluated the prevalence of NAFLD in patients with PCOS. One study evaluated 85 premenopausal women with PCOS and found that $55 \%$ of the study group had NAFLD, but $40 \%$ of women with NAFLD had normal body mass index. ${ }^{13}$ A recent meta-analysis also found that women with PCOS have four times higher risk of developing NAFLD compared with a healthy control group. ${ }^{14}$

IR, which is a key feature of metabolic syndrome, increases lipolysis in the adipose tissue, which leads to an increased stream of fatty acids to the liver and consequently hepatic fat accumulation. ${ }^{15}$ Visceral adipose tissue is an important regulator of fat accumulation in

\begin{tabular}{|c|c|c|c|c|}
\hline Variable & OR & LCl & $\mathrm{UCI}$ & $P$ value \\
\hline Race (compared with white) & & & & $<0.05$ \\
\hline Black & 0.52 & 0.51 & 0.53 & \\
\hline Hispanic & 1.12 & 1.10 & 1.13 & \\
\hline Asian or Pacific Islander & 0.68 & 0.66 & 0.70 & \\
\hline Native American & 1.06 & 1.01 & 1.13 & \\
\hline Other & 0.86 & 0.83 & 0.88 & \\
\hline Obesity & 2.79 & 2.7 & 2.82 & $<0.001$ \\
\hline Hypertension & 1.51 & 1.49 & 1.52 & $<0.001$ \\
\hline Dyslipidaemia & 1.50 & 1.48 & 1.51 & $<0.001$ \\
\hline Diabetes mellitus & 2.27 & 2.25 & 2.30 & $<0.001$ \\
\hline Hypothyroidism & 1.34 & 1.32 & 1.35 & $<0.001$ \\
\hline PCOS & 4.30 & 4.11 & 4.50 & $<0.001$ \\
\hline
\end{tabular}

$\mathrm{LCl}$, lower $\mathrm{Cl}$; PCOS, polycystic ovarian syndrome; UCl, upper $\mathrm{Cl}$. the liver determined by the flow of fatty acids through the portal vein. ${ }^{16} \mathrm{Up}$ to one-third of patients with liver steatosis will have inflammation and fibrosis in the liver, leading to NASH and consequently to cirrhosis. ${ }^{17} \mathrm{IR}$, obesity and oxidative stressors are reported by multiple studies as independent risk factors for inflammation and fibrosis development in patients with NAFLD. ${ }^{18-21}$

Although there is growing evidence on an association between PCOS and NAFLD, as they share similar metabolic comorbidities, the question remains whether androgen excess is a confounding factor for the development of NAFLD, in addition to a shared metabolic dysfunction. A large study in the $\mathrm{UK}^{22}$ evaluated 63125 women to assess the risk factors for NAFLD in women with PCOS from 2000 until 2016. In that study, free testosterone level and decreased sex hormone binding protein were associated with an increased risk of NAFLD. Another cross-sectional study of 400 Chinese women with PCOS found that the free androgen index, which reflects androgen bioavailability, was associated with increased risk of NAFLD. ${ }^{23}$ Another meta-analysis also suggested that high androgen levels are associated with NAFLD in women with PCOS. Furthermore, Jones $e t a l^{24}$ also suggested that women with PCOS and hyperandrogenism (HA) were more likely to have NAFLD compared with women with PCOS without HA, suggesting that elevated androgen levels contribute to NAFLD formation.

On the contrary, a few studies suggested that the androgen level did not increase the risk of NAFLD, but these studies were very limited in size. ${ }^{89}$

It is believed that androgen excess increases IR by affecting the insulin's effect on muscle and peripheral tissues, increasing visceral adipose tissue and reducing the adiponectin, the main insulin-sensitising hormone of adipose tissue. ${ }^{5}$ In addition, IR increases central adiposity, which increases the free fatty acids flow to the liver through the portal vein, which contributes to NAFLD formation. ${ }^{1016}$

Metformin is recommended for patients with PCOS with impaired fasting glucose or DM who do not respond to lifestyle modifications. ${ }^{24}{ }^{25}$ In addition, metformin decreases the total testosterone levels and the free testosterone index in patients with PCOS. ${ }^{26-28}$ Given the favourable metabolic effects of metformin, it is plausible to suggest that metformin may be effective in decreasing 
the risk of NAFLD development in PCOS, but no longterm clinical trials have been conducted to confirm this theory.

To our knowledge, this is the largest cross-sectional study that has evaluated the association between PCOS and NAFLD in a large segment of the US population. This association can be explained by the proposed role of androgen excess in NAFLD development in patients with PCOS. Due to ICD-9 limitations, NIS cannot specify if the diagnosis of NAFLD was biopsy-proven or based on imaging studies. Also, NIS cannot provide clinical information about specific medication use or laboratory results for each individual, including androgen level. In addition, NIS relies on the accuracy of clinical data and validity of medical diagnoses, which might be different between individuals and facilities. Because NIS is based on inpatient data, this inclusion could lead to more sick individuals in the data, which might affect the generalisability of the results. These factors could explain the lower prevalence of PCOS in our database compared with the general population. The exclusion of academic hospitals by the database could potentially exclude patients with more complex disease.

This large nationwide database study showed a strong and independent association between PCOS and NAFLD after adjusting for potential confounding factors including DM and obesity. This finding supports the recent evidence that suggests hyperandrogenism contributes to NAFLD formation in these patients. Further studies are needed to assess the efficacy of specific treatments for NAFLD in women with PCOS.

\section{Author affiliations \\ ${ }^{1}$ Gastroenterology and Hepatology, Medical College of Georgia, Augusta University, Augusta, Georgia, USA \\ ${ }^{2}$ Gastroenterology and Hepatology, Cleveland Clinic, Cleveland, Ohio, USA \\ ${ }^{3}$ Internal Medicine, Cleveland Clinic, Cleveland, Ohio, USA \\ ${ }^{4}$ Gastroenterology and Hepatology, Mayo Clinic, Rochester, Minnesota, USA}

Correction notice The article has been corrected since it is published. Author name, Yamen Ezaizi has been updated.

Contributors MMA: study concept, data interpretation, manuscript writing and editing. MTS: manuscript editing, data interpretation, critical revision. $\mathrm{FB}, \mathrm{YA}-\mathrm{K}, \mathrm{YE}$, SD: manuscript editing, critical revision. AM: study concept, data interpretation, manuscript editing.

Funding The study was funded in part by NIH R21 AR071046 (SD); R01 DK 113196 (SD); R01 GM119174 (SD); R56HL141744 (SD); P50 AA024333 (SD); U01 AA026976 (AM, SD); U01 DK061732 (AM, SD).

Competing interests None declared.

Patient consent for publication Not required.

Ethics approval Because NIS is a publicly available database, institutional board review approval was not required.

Provenance and peer review Not commissioned; externally peer reviewed.

Data availability statement Data are available in a public, open access repository. This cross-sectional study was conducted using the National Inpatient Sample (NIS) data from 2002 to 2014. The NIS is the largest all-payer inpatient database in the USA and contains a sample of over eight million inpatient hospitalisations each year, which represents approximately $20 \%$ of all discharges from all community hospitals participating in the Healthcare Cost and Utilization Project.
Open access This is an open access article distributed in accordance with the Creative Commons Attribution Non Commercial (CC BY-NC 4.0) license, which permits others to distribute, remix, adapt, build upon this work non-commercially, and license their derivative works on different terms, provided the original work is properly cited, appropriate credit is given, any changes made indicated, and the use is non-commercial. See: http://creativecommons.org/licenses/by-nc/4.0/.

\section{ORCID iDs}

Mohammad Maysara Asfari http://orcid.org/0000-0003-0059-9538

Muhammad Talal Sarmini http://orcid.org/0000-0002-4871-207X

\section{REFERENCES}

1 Younossi ZM, Stepanova M, Afendy M, et al. Changes in the prevalence of the most common causes of chronic liver diseases in the United States from 1988 to 2008. Clin Gastroenterol Hepatol 2011;9:524-30.

2 Kim JJ, Kim D, Yim JY, et al. Polycystic ovary syndrome with hyperandrogenism as a risk factor for non-obese non-alcoholic fatty liver disease. Aliment Pharmacol Ther 2017;45:1403-12.

3 Azziz R, Woods KS, Reyna R, et al. The prevalence and features of the polycystic ovary syndrome in an unselected population. J Clin Endocrinol Metab 2004;89:2745-9.

4 Norman RJ, Dewailly D, Legro RS, et al. Polycystic ovary syndrome. Lancet 2007;370:685-97.

5 Diamanti-Kandarakis E, Dunaif A, Background I. Insulin resistance and the polycystic ovary syndrome revisited: an update on mechanisms and implications. Endocr Rev 2012;33:981-1030.

6 Diamanti-Kandarakis E, Dunaif $A$. Insulin resistance and the polycystic ovary syndrome revisited: an update on mechanisms and implications. Endocr Rev 2012;33:981-1030.

7 Hart R, Norman R. Polycystic ovarian syndrome--prognosis and outcomes. Best Pract Res Clin Obstet Gynaecol 2006;20:751-78.

$8 \mathrm{Wu}$ J, Yao X-Y, Shi R-X, et al. A potential link between polycystic ovary syndrome and non-alcoholic fatty liver disease: an update meta-analysis. Reprod Health 2018;15:77.

9 Zhang J, Hu J, Zhang C, et al. Analyses of risk factors for polycystic ovary syndrome complicated with non-alcoholic fatty liver disease. Exp Ther Med 2018;15:4259-64.

10 Kauffman RP, Baker TE, Baker V, et al. Endocrine factors associated with non-alcoholic fatty liver disease in women with polycystic ovary syndrome: do androgens play a role? Gynecol Endocrinol 2010;26:39-46.

11 Després J-P, Lemieux I. Abdominal obesity and metabolic syndrome. Nature 2006;444:881-7.

12 Dawson AJ, Sathyapalan T, Smithson JAJ, et al. A comparison of cardiovascular risk indices in patients with polycystic ovary syndrome with and without coexisting nonalcoholic fatty liver disease. Clin Endocrinol 2014;80:843-9.

13 De Leo V, Musacchio MC, Cappelli V, et al. Genetic, hormonal and metabolic aspects of PCOS: an update. Reprod Biol Endocrinol 2016;14:38.

14 Gambarin-Gelwan M, Kinkhabwala SV, Schiano TD, Bodian C, et al. Prevalence of nonalcoholic fatty liver disease in women with polycystic ovary syndrome. Clin Gastroenterol Hepatol 2007;5:496-501.

15 Ramezani-Binabaj M, Motalebi M, Karimi-Sari H, et al. Are women with polycystic ovarian syndrome at a high risk of non-alcoholic fatty liver disease; a meta-analysis. Hepat Mon 2014;14:e23235.

16 Macut D, Boži I, Bjeki J, et al. Polycystic ovary syndrome and nonalcoholic fatty liver disease. Eur J Endocrinol 2017:145-58.

17 Vassilatou E. Nonalcoholic fatty liver disease and polycystic ovary syndrome. World J Gastroenterol 2014;20:8351-63.

18 Kleiner DE, Brunt EM, Van Natta M, Van NM, et al. Design and validation of a histological scoring system for nonalcoholic fatty liver disease. Hepatology 2005;41:1313-21.

19 Asfari MM, Niyazi F, Lopez R, et al. The association of nonalcoholic steatohepatitis and obstructive sleep apnea. Eur J Gastroenterol Hepatol 2017;29:1380-4.

20 Chalasani N, Deeg MA, Crabb DW, Ph D. Systemic levels of lipid peroxidation and its metabolic and dietary correlates in patients with nonalcoholic steatohepatitis. Am J Gastroenterol 2004;99:1497-502.

21 Paradis V, Perlemuter G, Bonvoust F, et al. High glucose and hyperinsulinemia stimulate connective tissue growth factor expression: a potential mechanism involved in progression to fibrosis in nonalcoholic steatohepatitis. Hepatology 2001;34:738-44.

22 Kumarendran B, O'Reilly MW, Manolopoulos KN, et al. Polycystic ovary syndrome, androgen excess, and the risk of nonalcoholic 
fatty liver disease in women: a longitudinal study based on a United Kingdom primary care database. PLoS Med 2018;15:e1002542-20.

23 Cai J, Wu CH, Zhang Y, et al. High-free androgen index is associated with increased risk of non-alcoholic fatty liver disease in women with polycystic ovary syndrome, independent of obesity and insulin resistance. Int J Obes 2017:41:1341-7.

24 Jones H, Sprung VS, Pugh CJA, et al. Polycystic ovary syndrome with hyperandrogenism is characterized by an increased risk of hepatic steatosis compared to nonhyperandrogenic PCOS phenotypes and healthy controls, independent of obesity and insulin resistance. J Clin Endocrinol Metab 2012;97:3709-16.
25 McCartney CR, Marshall JC, Syndrome PO. Clinical practice. polycystic ovary syndrome.. N Engl J Med 2016;375:54-64.

26 Costello M, Shrestha B, Eden J, et al. Insulin-sensitising drugs versus the combined oral contraceptive pill for hirsutism, acne and risk of diabetes, cardiovascular disease, and endometrial cancer in polycystic ovary syndrome. Cochrane Database Syst Rev 2007:CD005552.

27 Johnson NP. Metformin use in women with polycystic ovary syndrome. Ann Transl Med 2014;2:56.

28 Yousef M, Omran S. Metformin and polycystic ovary syndrome. Int $J$ Health Sci 2019;1:75-80. 